\title{
Zdeněk Hustý
}

Beitrag zu einem Satz von Ráb

Czechoslovak Mathematical Journal, Vol. 19 (1969), No. 4, 716-722

Persistent URL: http://dml.cz/dmlcz/100931

\section{Terms of use:}

(C) Institute of Mathematics AS CR, 1969

Institute of Mathematics of the Czech Academy of Sciences provides access to digitized documents strictly for personal use. Each copy of any part of this document must contain these Terms of use.

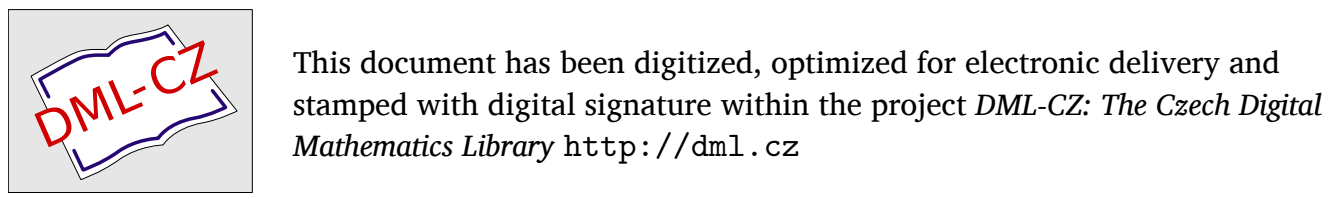




\title{
BEITRAG ZU EINEM SATZ VON RÁB
}

\author{
ZDENĚK HUSTÝ, Brno
}

(Eingelangt am 24. Februar 1969)

1. Das Symbol $O[f(x)]$, siehe $[1 ; 0.2,1]$, betrachten wir im Intervall $I=\left\langle x_{0}, \infty\right)$. Mit $\mathbf{M}\left[a_{i k}(x)\right]$ bezeichnet man eine quadratische Matrix von der Ordnung $n$, die in der $i$-ten Zeile und der $k$-ten Spalte das Element $a_{i k}(x)$ hat. Z. B. alle Elemente der Matrix $\mathbf{M}[a(x)]$ sind $a(x)$ gleich, $\mathbf{M}\left(\delta_{i k}\right)$ ist die Einheitsmatrix $\left(\delta_{i k}\right.$ ist das Kroneckersymbol). Es gelten folgende Beziehungen: $\sum_{s=1}^{m} \mathbf{M}\left[a_{i k}^{s}\right]=\mathbf{M}\left[\sum_{s=1}^{m} a_{i k}^{s}\right]$ (man kann auch $m=\infty$ wählen), $\int_{x_{0}}^{x} \mathbf{M}\left[a_{i k}(t)\right] \mathrm{d} t=\mathbf{M}\left[\int_{x_{0}}^{x} a_{i k}(t) \mathrm{d} t\right], O\left\{\mathbf{M}\left[a_{i k}(x)\right]\right\}=\mathbf{M}\left\{O\left[a_{i k}(x)\right]\right\}$, $\left|\mathbf{M}\left[a_{i k}(x)\right]\right|=\sum_{i, k=1}^{n}\left|a_{i k}(x)\right|$. Mit dem Symbol $\triangleright$ bezeichnet man eine einseitige Gleichheit. Z. B. ist $o(1) \triangleright O(1)$. Die umgekehrte Gleichheit gilt allgemein nicht.

Es sei $s=1,2, \ldots$ Die Schreibweise , $\mathbf{M}\left[a_{i k}^{s}(x)\right] \triangleright O\left\{\mathbf{M}\left[b_{i k}^{s}(x)\right]\right\}$ mit $C\left(a_{s}\right)^{\text {“c }}$ bedeutet, daß für alle $x \in I, i, k=1,2, \ldots, n$ die Ungleichungen $\left|a_{i k}^{s}(x)\right| \leqq C\left(a_{s}\right)$. - $\left|b_{i k}^{s}(x)\right|, 0<C\left(a_{s}\right) \in E_{1}$ gelten. Die Aussage , $\mathbf{M}\left[a_{i k}^{s}(x)\right] \triangleright O\left\{\mathbf{M}\left[b_{i k}^{s}(x)\right]\right\}$ gleichmä-

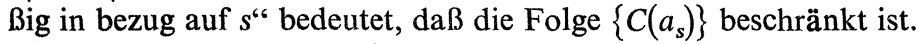

1.1. Es sei

$$
\mathbf{M}\left[a_{i k}(x)\right] \triangleright O\left\{\mathbf{M}\left[b_{i k}(x)\right]\right\}
$$

Dann ist

$$
\begin{gathered}
\left|\mathrm{M}\left[a_{i k}(x)\right]\right| \triangleright O\left\{\left|\mathrm{M}\left[b_{i k}(x)\right]\right|\right\}, \\
\int_{x_{0}}^{x}\left|\mathrm{M}\left[b_{i k}(t)\right]\right| \mathrm{d} t \triangleright O(1) \Rightarrow \int_{x_{0}}^{x}\left|\mathrm{M}\left[a_{i k}(t)\right]\right| \mathrm{d} t \triangleright O(1) .
\end{gathered}
$$

Beweis. Es gelte (1.1). Dann ist $\left|\mathbf{M}\left[a_{i k}(x)\right]\right|=\sum_{i, k=1}^{n}\left|a_{i k}(x)\right| \triangleright \sum_{i, k=1}^{n} O\left[b_{i k}(x)\right] \triangleright$ $\triangleright O\left\{\sum_{i, k=1}^{n}\left|b_{i k}(x)\right|\right\}$, so daß (1.2) in Kraft ist. Ferner ist $\int_{x_{0}}^{x}\left|\mathbf{M}\left[a_{i k}(t)\right]\right| \mathrm{d} t \triangleright$ $\triangleright \int_{x_{0}}^{x} O\left\{\left|\mathbf{M}\left[b_{i k}(t)\right]\right|\right\} \mathrm{d} t \triangleright O\left\{\int_{x_{0}}^{x}\left|\mathbf{M}\left[b_{i k}(t)\right]\right| \mathrm{d} t\right.$, so daß (1.3) gilt. 
1.2. Es sei $f(x)>0$ in $I, \int_{x_{0}}^{x} f(x) \mathrm{d} x \triangleright O(1)$. Ist $\mathbf{M}\left[a_{i k}(x)\right] \triangleright O\{\mathbf{M}[f(x)]\}$, so ist $\int_{x_{0}}^{x}\left|M\left[a_{i k}(t)\right]\right| \mathrm{d} t \triangleright O(1)$.

Beweis. Nach Abs. 1.1 genügt es zu zeigen, daß

$$
\int_{x_{0}}^{x}|\mathrm{M}[f(t)]| \mathrm{d} t \triangleright O(1) .
$$

Es ist

$$
\int_{x_{0}}^{x}|\mathbf{M}[f(t)]| \mathrm{d} t=\left|\mathbf{M}\left[\int_{x_{0}}^{x} f(t) \mathrm{d} t\right]\right| \triangleright|\mathbf{M}[O(1)]|=O(1) .
$$

1.3. Es sei $\mathbf{M}\left[a_{i k}(x)\right]$ eine positive Matrix in I. Folgende Beziehungen gelten

$$
\begin{gathered}
\int_{x_{0}}^{x} O\left\{\mathbf{M}\left[a_{i k}(t)\right]\right\} \mathrm{d} t=\int_{x_{0}}^{x} \mathbf{M}\left\{O\left[a_{i k}(t)\right]\right\} \mathrm{d} t=\mathbf{M}\left\{\int_{x_{0}}^{x} O\left[a_{i k}(t)\right] \mathrm{d} t\right\} \\
\triangleright \mathbf{M}\left\{O\left[\int_{x_{0}}^{x} a_{i k}(t) \mathrm{d} t\right]\right\}=O\left\{\mathbf{M}\left[\int_{x_{0}}^{x} a_{i k}(t) \mathrm{d} t\right]\right\}=O\left\{\int_{x_{0}}^{x} \mathbf{M}\left[a_{i k}(t)\right] \mathrm{d} t\right\} .
\end{gathered}
$$

Siehe z. B. [2; Seite 15-16]. Gilt (1.4), so sagen wir, daß die Symbole $\int_{x_{0}}^{x} O M$ vertauschbar sind. Ist $\int_{x_{0}}^{\infty} \mathbf{M}\left[a_{i k}(x)\right] \mathrm{d} x<\infty$, so sind auch die Symbole $\int_{x}^{\infty} O M$ vertauschbar.

1.4. Es seien $\mathbf{M}\left[b_{i k}^{s}(x)\right], s=1,2, \ldots$, positive Matrizen in $I$ und es sei $\mathbf{M}\left[a_{i k}^{s}(x)\right] \triangleright$ $\triangleright O\left\{\mathbf{M}\left[b_{i k}^{s}(x)\right]\right\}$ gleichmäßig in bezug auf $s$. Dann gilt

$$
\begin{gathered}
\sum_{s=1}^{\infty} \mathbf{M}\left[a_{i k}^{s}(x)\right] \triangleright \sum_{s=1}^{\infty} O\left\{\mathbf{M}\left[b_{i k}^{s}(x)\right]\right\}=\sum_{s=1}^{\infty} \mathbf{M}\left\{O\left[b_{i k}^{s}(x)\right]\right\}= \\
=\mathbf{M}\left\{\sum_{s=1}^{\infty} O\left[b_{i k}^{s}(x)\right]\right\}=\mathbf{M}\left\{O\left[\sum_{s=1}^{\infty} b_{i k}^{s}(x)\right]\right\}=O\left\{\mathbf{M}\left[\sum_{s=1}^{\infty} b_{i k}^{s}(x)\right]\right\}=O\left\{\sum_{s=1}^{\infty} \mathbf{M}\left[b_{i k}^{s}(x)\right]\right\},
\end{gathered}
$$

siehe $[2 ;$ S. 14$]$.

Ist ferner $\sum_{s=1}^{\infty} \mathbf{M}\left[b_{i k}^{s}(x)\right] \triangleright \mathbf{M}[O(1)]$, so ist $\sum_{s=1}^{\infty} \mathbf{M}\left[a_{i k}^{s}(x)\right] \triangleright \sum_{s=1}^{\infty} O\left\{\mathbf{M}\left[b_{i k}^{s}(x)\right]\right\}=$ $=O\left\{\sum_{s=1}^{\infty} \mathbf{M}\left[b_{i k}^{s}(x)\right]\right\} \triangleright O\{\mathbf{M}[O(1)]\}=\mathbf{M}[O(1)]$.

2. Wir führen folgende Bezeichnungen ein:

Die Funktionen

$$
z_{k}(x), \quad k=1,2, \ldots, n
$$

bzw.

$$
Z_{k}(x), \quad k=1,2, \ldots, n
$$


bilden ein Hauptsystem der Gleichung

$$
\begin{gathered}
z^{(n)}(x)+\sum_{i=1}^{n}\left(\begin{array}{l}
n \\
i
\end{array}\right) a_{i}(x) z^{(n-i)}(x)=0, \\
a_{i}(x) \in C_{0}(I), \quad i=1,2, \ldots, n
\end{gathered}
$$

bzw.

(p)

$$
\begin{gathered}
Z^{(n)}(x)=\sum_{i=1}^{n}\left(\begin{array}{l}
n \\
i
\end{array}\right)\left[a_{i}(x)+p_{i}(x)\right] Z^{(n-i)}(x)=0, \\
p_{i}(x) \in C_{0}(I), \quad i=1,2, \ldots, n .
\end{gathered}
$$

$\mathbf{M}\left[p_{i k}(x)\right]$ bezeichnet eine Matrix, deren Elemente folgendermaßen definiert sind:

$$
\begin{aligned}
& p_{i k}(x)=\left\langle\begin{array}{l}
0 \text { für } i=1,2, \ldots, n-1 ; \quad k=1,2, \ldots, n \\
-\left(\begin{array}{c}
n \\
n+1-k
\end{array}\right) p_{n+1-k}(x), \quad i=n, \quad k=1,2, \ldots, n .
\end{array}\right. \\
& \mathbf{M}\left[\varphi_{i k}(x)\right]=\mathbf{M}^{-1}\left[z_{k}^{(i-1)}(x)\right] \mathbf{M}\left[p_{i k}(x)\right] \mathbf{M}\left[z_{k}^{(i-1)}(x)\right],
\end{aligned}
$$

so daß

$$
\varphi_{i k}(x)=-\sum_{s=1}^{n}\left(\begin{array}{l}
n \\
s
\end{array}\right) p_{s}(x) \frac{W_{i}(x)}{W(x)}\left[z_{k}(x)\right]^{(n-s)}, \quad i, k=1,2, \ldots, n,
$$

wo $W(x)$ die Wronskische Determinante der Funktionen (2.1), und $W_{i}(x)$ das algebraische Komplement des Elementes $z_{i}^{(n-1)}(x)$ in der Determinante $W(x)$ darstellen.

3. Lemma. Ist

$$
\int_{x_{0}}^{\infty}\left|\mathbf{M}\left[\varphi_{i k}(x)\right]\right| \mathrm{d} x<\infty
$$

so ist

$$
\mathbf{M}\left[Z_{k}^{(i-1)}(x)\right]=\mathbf{M}\left[z_{k}^{(i-1)}(x)\right] \mathbf{M}\left[s_{i k}(x)\right],
$$

wo

gilt.

$$
\begin{gathered}
\mathbf{M}\left[s_{i k}(x)\right]=\sum_{s=0}^{\infty}(-1)^{s} \mathbf{M}\left[r_{i k}^{s}(x)\right], \\
\mathbf{M}\left[r_{i k}^{s}(x)\right]=\int_{x}^{\infty} \mathbf{M}\left[\varphi_{i k}(t)\right] \mathbf{M}\left[r_{i k}^{s-1}(t)\right] \mathrm{d} t, \\
s=1,2, \ldots, \quad \mathbf{M}\left[r_{i k}^{0}(x)\right]=\mathbf{M}\left(\delta_{i k}\right)
\end{gathered}
$$

Beweis. Wendet man die Resultate des Satzes von Ráb - siehe [3] - auf die Gleichung (p) an, so erhält man Lemma 3. 
4. Es gelte (3.1). Dann ist

$$
\mathbf{M}\left[r_{i k}^{s}(x)\right]=\int_{x}^{\infty} \int_{t_{1}}^{\infty} \ldots \int_{t_{s-1}}^{\infty} \mathbf{M}\left[\varphi_{i k}\left(t_{1}\right)\right] \ldots \mathbf{M}\left[\varphi_{i k}\left(t_{s}\right)\right] \mathrm{d} t_{s} \ldots \mathrm{d} t_{1},
$$

$s=1,2, \ldots$, wo $t_{0}=x \mathrm{zu}$ nehmen ist.

Die Formel (4.1) wird mittels Induktion in bezug auf $s$ mit Hilfe von (3.4) bewiesen.

5. Es gelten folgende Voraussetzungen:

$1^{\circ} f(x) \in C_{0}(I), 0<f(x) \triangleright O(1)$.

$2^{\circ} \mathbf{M}\left[f_{i k}(x)\right]>0, \mathbf{M}^{2}\left[f_{i k}(x)\right] \triangleright O\left\{\mathbf{M}\left[f_{i k}(x)\right]\right\}$ mit $C(f)$.

$3^{\circ}$ Die Matrizen $\mathbf{M}\left[f_{i k}(x) f^{s}(x)\right], s=1,2, \ldots$ sind monoton fallend.

$4^{\circ} x(x) \in C_{1}(I), x(x)>0, x^{\prime}(x)<0, \lim _{x \rightarrow \infty} x(x)=0$.

Ist

$$
\mathbf{M}\left[\varphi_{i k}(x)\right] \triangleright O\left(\mathbf{M}\left\{f_{i k}(x) f(x)\left[-x^{\prime}(x)\right]\right\}\right) \text { mit } C(\varphi),
$$

so gilt (3.1) und

$$
\mathbf{M}\left[r_{i k}^{s}(x)\right] \triangleright O\left(M\left\{f_{i k}(x) \frac{[f(x) x(x)]^{s}}{s !}\right\}\right) \text { mit } C(\varphi)^{s} C(f)^{s-1}, \quad s=1,2, \ldots
$$

Beweis. Es gelte (5.1). Es ist $\int_{x_{0}}^{x} f_{i k}(t) f(t)\left[-x^{\prime}(t)\right] \mathrm{d} t \triangleright O\left\{f_{i k}\left(x_{0}\right) f\left(x_{0}\right)\left[\varkappa\left(x_{0}\right)-\right.\right.$ $-\chi(x)]\} \triangleright O(1)$, so daß nach Abs. 1.2 die Ungleichung (3.1) gilt. Unter Berücksichtigung von (5.1) und Abs. 1.3 ist $\mathbf{M}\left[r_{i k}^{1}(x)\right]=\int_{x}^{\infty} \mathbf{M}\left[\varphi_{i k}(t)\right] \mathrm{d} t \triangleright O\left(\mathbf{M}\left\{\int_{x}^{\infty} f_{i k}(t)\right.\right.$. . $\left.\left.f(t)\left[-x^{\prime}(t)\right] \mathrm{d} t\right\}\right) \triangleright O\left\{\mathbf{M}\left[f_{i k}(x) f(x) \varkappa(x)\right]\right\}$ mit $C(\varphi)$, so daß (5.2) für $s=1$ gilt. Laut (3.4), (5.1), (5.2) ist $\mathbf{M}\left[r_{i k}^{s+1}(x)\right]=\int_{x}^{\infty} \mathbf{M}\left[\varphi_{i k}(t)\right] \mathbf{M}\left[r_{i k}^{s}(t)\right] \mathrm{d} t \triangleright \int_{x}^{\infty} O\left(\mathbf{M}\left\{f_{i k}(t)\right.\right.$. . $\left.\left.f(t)\left[-x^{\prime}(t)\right]\right\}\right) O\left(\mathbf{M}\left\{f_{i k}(t)\left([f(t) x(t)]^{s} / s !\right)\right\}\right) \mathrm{d} t \triangleright O\left(\mathbf{M}\left\{\int_{x}^{\infty} f_{i k}(t) f(t)^{s+1}\left(x(t)^{s} \mid s !\right)\right.\right.$. . $\left.\left.\left[-x^{\prime}(t)\right] \mathrm{d} t\right\}\right)$ mit $C(\varphi)^{s+1} . C(f)^{s}$. Nach den Voraussetzungen $3^{\circ}, 4^{\circ}$ ist $\mathbf{M}\left[r_{i k}^{s+1}(x)\right] \triangleright$ $\triangleright O\left\{M\left[f_{i k}(x)\left(f(x)(\chi(x))^{s+1} /(s+1) !\right]\right\}\right.$ mit $C(\varphi)^{s+1} . C(f)^{s}$, so daß (5.2) für alle $s=1,2, \ldots$ in Kraft ist.

6. Satz. Es gelten die Voraussetzungen des Abs. 5. Dann ist

$$
\mathbf{M}\left[Z_{k}^{(i-1)}(x)\right] \triangleright \mathbf{M}\left[z_{k}^{(i-1)}(x)\right]\left\{\mathbf{M}\left(\delta_{i k}\right)+\mathbf{M}\left[f_{i k}(x)\right] f(x) O[\varkappa(x)]\right\} .
$$

Beweis. Nach Abs. 5 gilt (3.1) und (5.2), so daß nach Lemma 3 die Formeln (3.2) und (3.3) gelten. Dann ist

$$
\mathbf{M}\left[s_{i k}(x)\right]=\mathbf{M}\left(\delta_{i k}\right)+\sum_{s=1}^{\infty}(-1)^{s} \mathbf{M}\left[r_{i k}^{s}(x)\right] .
$$


Laut (5.2) schließen wir, daß

$$
\mathbf{M}\left[r_{i k}^{s}(x)\right] \triangleright O\left\{\mathbf{M}\left(f_{i k}(x) \frac{[C f(x) \varkappa(x)]^{s}}{s !}\right)\right\}
$$

gleichmäßig in bezug auf $s$ gilt, wo $C=C(\varphi) . C(f)$ zu nehmen ist. Dann ist

$$
\begin{gathered}
\mathbf{M}\left[s_{i k}(x)\right] \triangleright \mathbf{M}\left(\delta_{i k}\right)+\sum_{s=1}^{\infty} o\left(\mathbf{M}\left\{f_{i k}(x) \frac{[C f(x) \varkappa(x)]^{s}}{s !}\right\}\right)=\mathbf{M}\left(\delta_{i k}\right)+ \\
+O\left(\mathbf{M}\left\{f_{i k}(x) \sum_{s=1}^{\infty} \frac{[C f(x) \varkappa(x)]^{s}}{s !}\right\}\right)=\mathbf{M}\left(\delta_{i k}\right)+O\left(\mathbf{M}\left\{f_{i k}(x)\left[e^{c f(x) x(x)}-1\right]\right\}\right) .
\end{gathered}
$$

Hieraus folgt wegen $1^{\circ}$

$$
\mathbf{M}\left[s_{i k}(x)\right] \triangleright \mathbf{M}\left(\delta_{i k}\right)+O\left\{\mathbf{M}\left[f_{i k}(x) f(x) x(x)\right]\right\} .
$$

Setzt man (6.2) in (3.2) ein, erhält man (6.1).

Bemerkungen. a) Anstatt (6.1) kann man mit Rücksicht auf (3.2), (5.2)

schreiben.

$$
\begin{aligned}
\mathbf{M}\left[Z_{k}^{(i-1)}(x)\right] & \triangleright \mathbf{M}\left[z_{k}^{(i-1)}(x)\right]\left\{\mathbf{M}\left(\delta_{i k}\right)+\sum_{s=1}^{j}(-1)^{s} \mathbf{M}\left[r_{i k}^{s}(x)\right]+\right. \\
& \left.+\mathbf{M}\left[f_{i k}(x)\right] f(x)^{j+1} O\left[x(x)^{j+1}\right]\right\}
\end{aligned}
$$

b) Die Voraussetzungen $1^{\circ}-3^{\circ}$ werden von den Funktionen $f_{i k}(x)=f(x)=1$ erfüllt, $i, k=1,2, \ldots, n$

7. Anstatt (6.1) resp. (6.3) kann man

$$
\begin{gathered}
Z_{k}^{(i-1)}(x) \triangleright z_{k}^{(i-1)}(x)+\sum_{v=1}^{n} z_{v}^{(i-1)}(x) f_{v k}(x) f(x) O[\chi(x)], \\
i, k=1,2, \ldots, n,
\end{gathered}
$$

resp.

$$
\begin{gathered}
Z_{k}^{(i-1)}(x) \triangleright z_{k}^{(i-1)}(x)+\sum_{s=1}^{j}(-1)^{s} \sum_{v=1}^{n} z_{v}^{(i-1)}(x) \sum_{v_{1}=1}^{n} \ldots \\
\ldots \sum_{v_{s}-1=1}^{n} \int_{x}^{\infty} \int_{t_{1}}^{\infty} \ldots \int_{t_{s-1}}^{\infty} \varphi_{v v_{1}}\left(t_{1}\right) \varphi_{v_{1} v_{2}}\left(t_{2}\right) \ldots \varphi_{v_{s-1} k}\left(t_{s}\right) \mathrm{d} t_{s} \ldots \mathrm{d} t_{1}+ \\
+\sum_{v=1}^{n} z_{v}^{(i-1)}(x) f_{v k}(x) f(x)^{j+1} O\left[x(x)^{j+1}\right], \quad i, k=1,2, \ldots, n
\end{gathered}
$$

schreiben, wo $v_{0}=v, t_{0}=x$ zu nehmen ist. 
8. Es existieren derartige positive Funktionen $P_{s}(x), s=1,2, \ldots, n$, daß für alle $i, k=1,2, \ldots, n$

$$
-\frac{W_{i}(x)}{W(x)}\left[z_{k}(x)\right]^{(n-s)} \triangleright O\left[f_{i k}(x) f(x) P_{s}(x)\right], \quad s=1,2, \ldots, n
$$

gilt, wobei die Funktionen $f_{i k}(x), f(x)$ die Voraussetzungen $1^{\circ}-3^{\circ}$ des Abs. 5 erfüllen. Gilt

$$
\int_{x_{0}}^{\infty}\left|p_{s}(x)\right| P_{s}(x) \mathrm{d} x<\infty, \quad s=1,2, \ldots, n,
$$

so hat die Gleichung (p) das Hauptsystem (7.1) resp. (7.2), wo

$$
\chi(x)=\int_{x}^{\infty} \sum_{s=1}^{n}\left(\begin{array}{l}
n \\
s
\end{array}\right)\left|p_{s}(t)\right| P_{s}(t) \mathrm{d} t
$$

gesetzt wird.

Dieses folgt aus Satz 6, denn nach den Formeln (2.3), (8.1)-(8.3) gilt $\mathbf{M}\left[\varphi_{i k}(x)\right] \triangleright$ $\triangleright O\left(M\left\{f_{i k}(x) f(x)\left[-x^{\prime}(x)\right]\right\}\right)$, wo die Funktion $x(x)$ der Voraussetzung $4^{\circ}$ des Abs. 5 entspricht.

9. Beispiel. Es sei (a) eine Gleichung mit konstanten Koeffizienten, deren charakteristische Gleichung nur einfache Wurzeln $\lambda_{i}=1,2, \ldots, n$ hat, wobei

$$
\operatorname{Re} \lambda_{1} \leqq \operatorname{Re} \lambda_{2} \leqq \ldots \leqq \operatorname{Re} \lambda_{n}
$$

gilt. Dann bilden die Funktionen $e^{\lambda_{k} x}, k=1,2, \ldots, n$ ein Hauptsystem der Gleichung (a). Es ist $\left[W_{i}(x) / W(x)\right] z_{k}^{(n-s)}(x)=Q_{i} \lambda_{k}^{n-s} \exp \left\{\left(\lambda_{k}-\lambda_{i}\right) x\right\}, i, k=1,2, \ldots, n$ mit

$$
\begin{aligned}
& Q_{i}=\prod_{\substack{j=1 \\
j \neq i}}^{n}\left(\lambda_{i}-\lambda_{j}\right)^{-1} \text {. Dann ist nach }(2.3) \\
& \qquad \varphi_{i k}(x)=-Q_{i} \exp \left\{\left(\lambda_{k}-\lambda_{i}\right) x\right\} \sum_{s=1}^{n}\left(\begin{array}{l}
n \\
s
\end{array}\right) p_{s}(x) \lambda_{k}^{n-s}= \\
& =-Q_{i} \exp \left\{\left(\lambda_{k}-\lambda_{i}+\lambda_{1}-\lambda_{n}\right) x\right\} \sum_{s=1}^{n}\left(\begin{array}{l}
n \\
s
\end{array}\right) p_{s}(x) \lambda_{k}^{n-s} \exp \left\{\left(\lambda_{n}-\lambda_{1}\right) x\right\} .
\end{aligned}
$$

Ist

$$
\int_{x_{0}}^{\infty}\left|p_{s}(x)\right| e^{\operatorname{Re}\left(\lambda_{n}-\lambda_{1}\right) x} \mathrm{~d} x<\infty, \quad s=1,2, \ldots, n,
$$

so gilt

$$
\varphi_{i k}(x) \triangleright O\left\{f_{i k}(x) f(x)\left[-\varkappa^{\prime}(x)\right]\right\}, \quad i, k=1,2, \ldots, n
$$


Wo

$$
\begin{gathered}
f_{i k}(x)=e^{\operatorname{Re}\left(\lambda_{k}-\lambda_{i}\right) x}, \quad f(x)=e^{\operatorname{Re}\left(\lambda_{1}-\lambda_{n}\right) x}, \\
x(x)=\int_{x}^{\infty} e^{\operatorname{Re}\left(\lambda_{n}-\lambda_{1}\right) t} \sum_{s=1}^{n}\left(\begin{array}{l}
n \\
s
\end{array}\right)\left|p_{s}(t)\right| \mathrm{d} t
\end{gathered}
$$

gesetzt wird. Die Funktionen (9.4) in bezug auf (9.1), (9.2) erfüllen die Voraussetzungen $1^{\circ}-4^{\circ}$ des Abs. 5 und nach (9.3) gilt (5.1) mit $C(\varphi)=Q \Lambda^{n}$, wo $Q=\max$. . $\left\{\left|Q_{1}\right|, \ldots,\left|Q_{n}\right|\right\}, \Lambda=\max \left\{1,\left|\lambda_{1}\right|,\left|\lambda_{2}\right|, \ldots,\left|\lambda_{n}\right|\right\}$ ist. Gemä $\beta$ dem Satz 6 und der Formel (7.2), wo $j=1$ gesetzt wird, hat die Gleichung (p) das Hauptsystem

$$
\begin{gathered}
Z_{k}^{(i-1)}(x) \triangleright e^{\lambda_{k} x}\left\{\lambda_{k}^{i-1}+\sum_{v=1}^{n} \lambda_{v}^{i-1} \int_{x}^{\infty} e^{\left(\lambda_{k}-\lambda_{v}\right)(t-x)} \psi_{v k}(t) \mathrm{d} t+e^{2 \operatorname{Re}\left(\lambda_{1}-\lambda_{n}\right) x} O\left[\chi^{2}(x)\right]\right\}, \\
i, k=1,2, \ldots, n,
\end{gathered}
$$

wo

$$
\psi_{v k}(x)=Q_{v} \sum_{s=1}^{n}\left(\begin{array}{l}
n \\
s
\end{array}\right) \lambda_{k}^{n-s} p_{s}(x)
$$

zu nehmen ist. Dieses Ergebnis wird in der Arbeit [4] angeführt.

\section{Literatur}

[1] Z. Hustý: Asymptotische Eigenschaften der Differentialgleichung $y^{\prime \prime}+2 a_{1}(x) y^{\prime}+a_{2}(x) y=$ = 0. Czechoslovak Math. J. 19(94) (1969) 208-240.

[2] A. Erdélyi: Asymptotic expansions. Russische Übersetzung. Moskva 1962.

[3] M. Ráb: Note sur les formules asymptotiques pour les solutions d'un système d'équations différentielles linéaires. Czechoslovak Math. J. 16 (91) (1966), 127-129.

[4] L. Konečný: O aplikaci jedné Rábovy věty na jistou perturbovanou diferenciální rovnici $n$-tého řádu. Im Druck.

Anschrift des Verfassers: Brno, Zemědělská 5, ČSSR (Vysoká škola zemědělská). 